\title{
Saragosse et les danseurs de Lausanne
}

\section{François Rosset}

\section{OpenEdition}

\section{Journals}

Édition électronique

URL : http://journals.openedition.org/edl/448

DOI : $10.4000 /$ edl. 448

ISSN : 2296-5084

\section{Éditeur}

Université de Lausanne

\section{Édition imprimée}

Date de publication : 15 décembre 2012

Pagination : 7-14

ISBN : 978-2-940331-29-1

ISSN : 0014-2026

\section{Référence électronique}

François Rosset, «Saragosse et les danseurs de Lausanne », Études de lettres [En ligne], 4 | 2012, mis en ligne le 15 décembre 2015, consulté le 19 décembre 2020. URL : http://journals.openedition.org/ edl/448 ; DOI : https://doi.org/10.4000/edl.448 


\section{SARAGOSSE ET LES DANSEURS DE LAUSANNE}

Les lectrices, les lecteurs d'Etudes de Lettres ne sont sans doute pas tous familiers de l'objet qui est au centre des travaux réunis dans la présente livraison. Deux mots de présentation ne seront peut-être pas inutiles.

Le Manuscrit trouvé à Saragosse est un roman écrit entre 1794 et 1813 ou 1814 par Jean Potocki, aristocrate polonais, historien, voyageur, érudit profond et original, grand seigneur dont la vie fournirait elle-même la trame du plus alambiqué des romans d'aventures ${ }^{1}$. Tout aussi agitée et pleine de surprise est l'histoire du texte lui-même, écrit en français (comme l'ensemble de la production de Potocki: récits de voyage, théâtre, textes politiques, ouvrages historiques), jamais publié pleinement du vivant de l'auteur, connu d'abord par une traduction polonaise parue en 1847, redécouvert en France en 1958 à la faveur d'une édition fragmentaire due à Roger Caillois, devenu populaire grâce au film admirable qu'en a donné Jerzy Has en 1964, édité en 1989 sous une forme annoncée comme complète, mais assez bancale en réalité, donnée enfin à lire pour la première fois sous les deux versions réellement écrites par l'auteur, en 2006, puis en $2008^{2}$. Ainsi, l'histoire du Manuscrit trouvé à Saragosse inclut à peu près tout ce qui peut arriver à un texte: écriture et réécritures, disparition et réapparition, traductions, copies, mutilations,

I. Pour une présentation de cette vie étonnante, voir F. Rosset, D. Triaire, Jean Potocki. Biographie.

2. Les deux auteurs de la biographie ont aussi publié une édition des Euvres de Potocki, dont les volumes IV-1 et IV-2 comportent les versions de 1804 et de $1810 \mathrm{du}$ Manuscrit trouvé à Saragosse (Louvain, Peeters, 2004-2006, cinq tomes en six volumes). Les deux versions du roman ont été republiées, avec un nouvel apparat critique, dans la collection GF-Flammarion en 2008. Toutes les citations du roman incluses ci-après se réfèrent à cette dernière édition. 
manipulations, plagiats, transpositions, pastiches, etc., autant d'opérations qui sont d'ailleurs racontées, d'une manière ou d'une autre, à l'intérieur même du roman.

Le contenu de ce dernier comme sa forme ne sont pas moins curieux. Il n'est pas exagéré de dire qu'on y trouve, sur ces deux plans, une somme de tout ce que la tradition narrative occidentale a pu charrier jusqu'à la grande redistribution des terres et des idées en 1815 (moment que Potocki choisit, très significativement, pour se donner la mort). Tissant un réseau déroutant de récits, Potocki revisite, dans le ton de cette ironie tout à la fois légère et ravageuse qui lui est propre, les croyances, les théories et discours d'explication du monde, les lieux communs ainsi que les figures et la topique qui balisent l'espace du roman européen, les modèles de récits et de collections de récits. Un livre qui ponctue en quelque sorte la lignée portée par Cervantes, Swift, Sterne, le Diderot de Jacques le Fataliste et son maître, le Goethe du Wilhelm Meister; mais une œuvre qui est insuffisamment connue encore, compte tenu de sa réelle importance, de sa richesse comme de l'étonnante expérience de lecture qu'elle offre, et en dépit du fait qu'elle jouit dans des milieux fort divers du statut de livre-culte.

Les travaux consacrés à cette œuvre et à son auteur se sont multipliés ces dernières années, après avoir dépendu trop longtemps de l'enthousiasme de quelques pionniers (Leszek Kukulski, Maria Ewelina Zoltowska, Daniel Beauvois) ${ }^{3}$. Outre les éditions déjà citées, des revues d'audience internationale lui ont dédié des numéros spéciaux ${ }^{4}, \mathrm{~d}^{\prime} \mathrm{im}$ portants ouvrages collectifs ont paru dans différents pays ${ }^{5}$, deux thèses viennent d'être soutenues avec brio ${ }^{6}$ et cinq autres sont en cours (à Montpellier, à Lausanne, à Neuchâtel, à Berlin).

3. Une bibliographie mise à jour de ces travaux, ainsi que d'autres informations sont accessibles sur le site http://recherche.univ-montp3.fr/jeanpotocki/.

4. Les Cahiers de l'Association Internationale des Etudes Françaises en 1999, Europe en 2001, Comparative Criticism en 2002.

5. J. Herman, P. Pelckmans, F. Rosset (éds), Le Manuscrit trouvé à Saragosse et ses intertextes; E. Jastrzebowska, M. Niewojt (éds), Archeologia, Letteratura, Collezionismo; F. Rosset, D. Triaire (éds), Jean Potocki ou le dédale des Lumières; E. Klene (éd.), Jean Potocki à nouveau; K. Joucaviel (éd.), Jean Potocki.

6. Lorenz Frischknecht, Quand le comment explique le pourquoi: les trois versions successives du Manuscrit trouvé à Saragosse $(1794,1804,18010)$ de Jean Potocki, Université de Montpellier III et de Zurich, 2012; Marius Warholm Haugen, Jean Potocki: esthétique et philosophie de l'errance, Université de Trondheim, 2012. 
Mais ce qu'il faut dire surtout, c'est que cette vitalité de la recherche ne cesse d'enrichir notre connaissance de l'œuvre de Potocki. Depuis quelques années, les découvertes se multiplient dans les archives de Russie, d'Ukraine, de Pologne et d'ailleurs, mettant au jour des textes inconnus jusque-là, des lettres, des documents de diverses natures; on peut s'attendre à d'autres trouvailles qui justifieront sans doute, d'ici quelques années, une sérieuse révision de nos connaissances et de nos convictions actuelles.

Parfois, ce sont des trouvailles d'ampleur minuscule qui permettent de conforter des points de vue ou qui fournissent de nouvelles images, de nouvelles potentialités métaphoriques pour exprimer des idées de lecteur ou pour décrire les activités individuelles et collectives des chercheurs. Prenons le cas de cette feuille manuscrite conservée au Musée historique de Lausanne. C'est un document qui n'a l'air de rien. Une liste. Elle comporte les quarante-quatre noms des souscripteurs qui ont payé les deux louis neufs requis pour être admis à danser une fois par semaine dans la salle de M. de Mézery, au Chêne, à Lausanne ${ }^{7}$. Nous sommes probablement en 1776, la bonne société s'amuse comme elle peut, accueillant de nombreux étrangers plus ou moins fortunés qui s'arrêtent pour quelques années ou quelques jours, c'est selon. Beaucoup d'Anglais, des Allemands, des Français, des Polonais, des Russes. Sur la liste figure celui que beaucoup sont venus consulter, le docteur Tissot, quelquesuns des notables du lieu (on remarque au milieu d'eux un dénommé F. Rosset), et plusieurs de ces hôtes de passage; des vieillards, des jeunes, des enfants. Parmi ceux-ci, le comte J. Potocki et le comte S. Potocki: Jean et Séverin, âgés de quinze et quatorze ans, qui séjournent dans la région depuis deux bonnes années sous la gouverne du pasteur Louis Constançon, leur mentor. Des ordres lui ont sans doute été donnés par la mère des deux garçons, figure remarquée de la haute société de l'Europe: les humanités et les sciences, certes, quelques rudiments de théologie, à la rigueur, mais surtout ne pas négliger la vie sociale où l'on ne saurait, quand on est né si bien pourvu, faire triste figure. Et peaufiner cette science-là, même loin des palais de Vienne et de Varsovie, vaut bien trois fois deux louis à répertorier dans la colonne des dépenses diverses.

7. Musée historique de Lausanne, Fonds Bridel, carton 56 (merci à Béatrice Lovis de m’avoir communiqué ce document). 
Liste de souscripteurs pour le bal au Grand Chêne (XVIII siècle).

Fonds Bridel, carton 56 (fiche Museris: Bridel.07.01.001.003). 
Les éléments livrés par ce document sont bien propres à remuer l'imagination; tant de questions restent suspendues: les garçons ont-ils réellement participé à ces sauteries hebdomadaires? qui y fréquentaientils en particulier? quelles amitiés se sont-elles nouées? furent-ils lassés ou divertis par ces rituels de provinciale sociabilité? En réalité, c'est dans le roman qui sera écrit vingt ans plus tard que pointe, faute de réponses à ces questions, une lumière latérale sur ces scènes lausannoises dont on ne sait pratiquement rien.

Ils sont aussi deux frères, nés dans l'une des plus grandes maisons de l'Espagne, Henrique et Carlos Velasquez. L'un a le génie du calcul, l'autre n'a de goût que pour les futiles amusements; un ingénieur hors pair et le premier des talons rouges. Mais leurs destins se mélangent: le hasard donnera au premier la vie qui était promise au second. Celui-ci en déduira tristement que l'art de la sarabande est décidément la seule recette du succès dans un monde si désordonné. Telle est l'histoire racontée par le géomètre Velasquez, le fils du malheureux Henrique, qui remplit quelques dizaines de pages mémorables du Manuscrit trouvé à Saragosse. La comtesse Anne-Thérèse Potocka, née Ossolinska, n'avait peut-être pas tort de faire danser ses fils, ne fût-ce qu'à Lausanne.

Quoi qu'il en soit, l'important, comme dans la danse, c'est d'être deux; car ils sont toujours deux, les personnages du roman de Potocki, tous soumis au régime d'une binarité qui combine dédoublement, duplication et duplicité, substitution, quiproquo. Chez les Velasquez, c'est le mode du contraste qui s'impose. L'hypertrophie de l'intellect génère les inventions les plus remarquables, tout en occasionnant les scènes les plus grotesques; en face, l'attirance du grand monde permet de célébrer l'éclat des cours, avant d'en expérimenter amèrement la vanité. Mais comme on le voit, l'anecdotique trajectoire des individus prend du sens quand elle est conduite à croiser celle des autres et quand de cette rencontre émane une figure, un modèle, un régime. C'est ainsi que le Manuscrit trouvé à Saragosse multiplie les actualisations narratives de l'imitation et de la copie, de la différence et de l'identité, du dialogue et du dialogisme, autant de retombées discursives, morales et esthétiques du binarisme dominant.

C'est à cette contrainte majeure qu'est soumise la lecture de ce roman si particulier. Une même panoplie d'instruments n'est jamais suffisante. D'autres voix, d'autres points de vue, d'autres a priori sont toujours nécessaires, de la même façon que le Manuscrit lui-même ne cesse de 
nous dire qu'une même histoire n'a jamais qu'une seule occurrence, une seule forme, une seule incarnation. Et que son sens, par conséquent, se diffracte dans la multiplicité des possibles; il pourrait même en aller pareillement pour les systèmes de croyances et de valeurs qui soustendent l'établissement du sens. Prétendre circonscrire la signification de ce roman, chercher à en démontrer la vérité, c'est, comme le fait le héros du roman, descendre dans la mine d'or des Gomelez, pour constater finalement que la veine s'est épuisée, non pas parce qu'elle aurait subi l'inexorable dissolution dans le néant, mais parce qu'elle a distribué l'entier de ses ressources, tous azimuts et sous toutes formes. La découverte récente de la déclinaison successive du roman lui-même en plusieurs versions confirme tout cela à l'échelle macroscopique de l'œuvre.

C'est aussi à cela que tient le projet concrétisé par le dossier réuni ici. Il y eut d'abord, comme il y a plus de deux siècles, une manière de souscription à Lausanne. Les cotisants ont payé leur tribut en participant activement à une journée d'étude qui s'est tenue le 27 janvier 2010 et qui visait justement à faire entendre des voix venues d'ailleurs, ancrées dans des contextes épistémologiques et méthodologiques différents. Diverses spécialités ont été mobilisées pour soumettre le Manuscrit trouvé à Saragosse à une pluralité de lectures. Lire Potocki en latiniste (Danielle van Mal-Maeder), en médiéviste (Alain Corbellari), en russisant (Leonid Heller), en arabisant (Richard van Leeuwen), en philosophe (Philippe Grosos), en historienne du discours naturaliste (Nathalie Vuillemin), en physicien (Maxime Gabella), en historien de l'art (Jan Blanc) - tel fut le pari relevé en présence de quelques doctorants attentifs, qui ont été invités à apporter aussi leur tribut à la présente publication (Emilie Klene, Lena Seauve, Lorenz Frischknecht). Et si F. Rosset est inscrit sur la liste des danseurs lausannois depuis longtemps, il ne pouvait pas manquer non plus Dominique Triaire qui apporte au bouquet des contributions une senteur piquante d'inspiration biographique.

François Rosset Université de Lausanne 


\section{BIBLIOGRAPHIE}

Herman, Jan, Pelckmans, Paul, Rosset, François (éds), Le Manuscrit trouvé à Saragosse et ses intertextes. Actes du colloque international, Leuven-Anvers, 30 mars - $1^{\text {er }}$ avril 2000, Louvain, Peeters, 2001. Jastrzebowska, Elzbieta, Niewojt, Monika (edd.), Archeologia, Letteratura, Collezionismo. Atti del Convegno dedicato a Jan e Stanislaw Kostka Potocki, Roma, Accademia Polacca delle Scienze, 2008.

Joucaviel, Kinga (éd.), Jean Potocki: pérégrinations, Toulouse, Presses universitaires du Mirail, 2012.

Klene, Emilie (éd.), Jean Potocki à nouveau, Amsterdam, Rodopi, 2010. Rosset, François, Triaire, Dominique, Jean Potocki. Biographie, Paris, Flammarion, 2004.

- (éds), Jean Potocki ou le dédale des Lumières, Montpellier, Presses universitaires de la Méditerranée, 2010.

\section{Crédit iconographique}

Page 10 :

(C) Musée historique de Lausanne. Scan: atelier de numérisation de la Ville de Lausanne. 
